\title{
DIVERSIDAD Y COMPOSICIÓN DE PLANTAS VASCULARES EN HUMEDALES DEL PÁRAMO RABANAL, BOYACÁ- COLOMBIA
}

\author{
DIVERSITY AND COMPOSITION OF VASCULAR PLANTS IN \\ WETLANDS OF THE PARAMO RABANAL, BOYACÁ- COLOMBIA
}

\author{
1 Magda-Juliana Cuello-Salinas \\ 2 Manuel Galvis-Rueda
}

\begin{abstract}
${ }^{1}$ Msc. en Ciencias Biológicas. Grupo de Estudios en Ecología, Etología, Educación y Conservación (GECOS). Universidad Pedagógica y Tecnológica de Colombia, Tunja, Colombia

2Msc. en Educación. Grupo de Estudios en Ecología, Etología, Educación y Conservación (GECOS). Universidad Pedagógica y Tecnológica de Colombia, Tunja, Colombia

${ }^{1}$ magda.cuello@uptc.edu.co; ${ }^{2}$ manuel.galvis@uptc.edu.co
\end{abstract}

\section{RESUMEN}

Contextualización: El páramo de Rabanal posee humedales que proveen el recurso hídrico, indispensable para el desarrollo de la economía y de la seguridad alimentaria de la región. Este ecosistema está siendo alterado por la presión que ejercen las diversas actividades antrópicas productivas y económicas, por lo cual, el humedal pierde la capacidad para retener y almacenar agua, facilitando la expansión de especies exóticas que aumentan la sedimentación, reduciendo los niveles de retención y almacenamiento de agua, degradando el suelo y evitando el crecimiento de especies nativas. La vegetación asociada permite la regulación del ciclo hidrológico y favorece una medida de adaptación al clima cambiante.

Vacío de investigación: en los humedales del páramo Rabanal existe escasa información sobre la influencia de la vegetación asociada a los cuerpos de agua que contribuyen a su conservación.
Propósito del estudio: identificar la flora vascular presente en las zonas circundantes a los humedales artificiales del páramo Rabanal.

Metodología: se realizó la caracterización, composición y estructura de la vegetación vascular en zonas circundantes de los embalses Teatinos, Gachaneca I y II en el páramo Rabanal, en municipios de Ventaquemada y Samacá, Boyacá, en cinco salidas de campo se hizo el levantamiento de 32 parcelas dependiendo de las características fisionómicas de la vegetación siguiendo un diseño anidado. El material vegetal se procesó en el Herbario de la Universidad Pedagógica y Tecnológica de Colombia de Tunja (UPTC); la determinación se hizo con el apoyo de claves taxonómicas, bases de datos y expertos. En términos de riqueza, la diversidad se comparó entre los embalses mediante un Análisis de Varianza en rangos o prueba de Kruskall-Wallis. 
Resultados y conclusiones: la mayor riqueza de especies de Margalef se presentó en la parcela P2 $(5,40)$, la mayor dominancia de Simpson fue para la parcela P2 $(0,95)$, la mayor diversidad de Shannon corresponde a la parcela P2 $(4,39)$ del Embalse Teatinos, el índice de similaridad de Sörensen indica que los embalses Gachaneca I y II, poseen un $52,63 \%$ de similaridad. La evidencia de actividades antrópicas productivas y económicas provoca una pérdida de la vegetación nativa y una fragmentación del ecosistema.

Palabras clave: composición; diversidad; embalses; estructura; riqueza; paramo; comunidades vegetales.

\section{ABSTRACT}

Contextualization: The Paramo of Rabanal has wetlands that provide the water resource, indispensable for the development of the economy and food security of the region. This ecosystem is being altered by the pressure exerted by the various productive and economic human activities, which means that the wetland loses the capacity to retain and store water, facilitating the expansion of exotic species that increase sedimentation, reducing water retention and storage levels, degrading soil and preventing the growth of native species. The associated vegetation allows the regulation of the hydrological cycle and promotes a measure of adaptation to the changing climate.

Knowledge gap: there is little information on the influence of vegetation associated with water bodies that contribute to their conservation in the wetlands of the Rabanal paramo.
Purpose: identify the vascular flora present in the areas surrounding the artificial wetlands of the Rabanal paramo.

Methodology: the characterization, composition and structure of vascular vegetation was carried out in the areas surrounding the Teatinos, Gachaneca I and II reservoirs in the Rabanal paramo, in the municipalities of Ventaquemada and Samacá, Boyacá, in five field trips was made the survey of 32 plots depending on the physical characteristics of the vegetation following a nested design. The plant material was processed at the Herbarium of the Pedagogical and Technological University of Colombia, Tunja (UPTC); the determination was made with the support of taxonomic keys, databases and experts. In terms of richness, diversity was compared between reservoirs using a Variance Analysis in ranges or Kruskall-Wallis test.

Results and conclusions: The greatest richness of Margalef species was presented in plot P2 $(5,40)$, the greatest dominance of Simpson was for plot P2 $(0,95)$, the greatest diversity of Shannon corresponds to plot P2 $(4,39)$ of the Teatinos Reservoir, the similarity index of Sörensen indicates that the reservoirs Gachaneca I and II, have 52,63\% similarity. Evidence of productive and economic anthropic activities leads to loss of native vegetation and fragmentation of the ecosystem.

Key words: composition; diversity; reservoirs; structure; wealth; paramo; plant communities. 


\section{RESUMEN GRÁFICO}

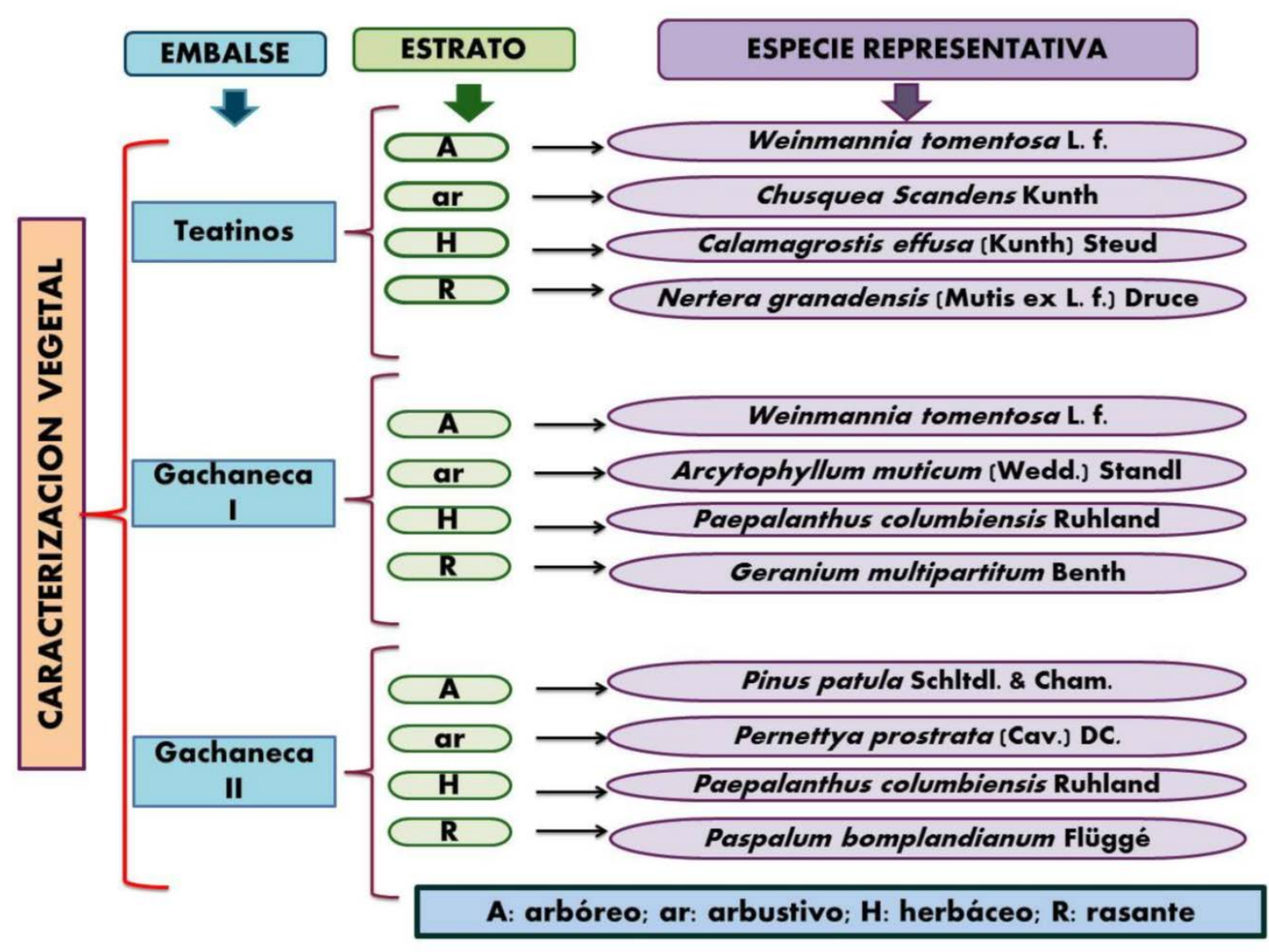

Fuente: Autores.

\section{INTRODUCCIÓN}

Los páramos son regiones desarboladas y frías (oscila entre $2-10^{\circ} \mathrm{C}$ ) situadas en la parte más alta de las montañas, entre el límite superior del bosque alto andino (3.200-3.800 $\mathrm{m}$ de altitud) y el límite inferior de las nieves perpetuas (4.400-4.700 m de altitud) (Cuatrecasas, 1989), son considerados como biomas exclusivos de las montañas neotropicales que se distribuyen a manera de islas sobre las cadenas montañosas de Costa Rica, Panamá y los Andes de Venezuela, Colombia, Ecuador y el norte de Perú (Cleef 1978, Molano 1989, Luteyn 1999). Las altas montañas andinas sobresalen por la calidad de sus paisajes, la gran diversidad de formas de vida que allí persisten y las importantes funciones ecológicas que presentan (Tapia, 2014). Los páramos se destacan por su oferta de servicios ecosistémicos, como la conservación de la biodiversidad, la provisión de hábitats para distintas especies, la regulación hídrica, la absorción de carbono (Buytaert et al., 2007). Los páramos poseen un conjunto muy característico de humedales de gran importancia para las comunidades humanas que han habitado en sus áreas de influencia para el desarrollo de las poblaciones (Etter, McAlpine y Possingham, 2008).

La población cada vez más depende del páramo directa o indirectamente, lo que ha generado disturbios en estos ecosistemas los últimos años, diferentes actividades como 
minería, forestación con especies exóticas, construcción de vías, acueductos, sistemas de drenajes y represas, lo han convertido en un ecosistema amenazado por el incremento de presión sobre los recursos naturales que posee (Gomezcoello, 2020). Estas actividades afectan directamente la estructura del suelo de paramo y de los servicios ecosistémicos que proporciona, y por ende produce la reducción de la cobertura vegetal, afectando la zona de captación de las principales microcuencas y la capacidad amortiguadora de la vegetación (CAR y Corpochivor, 2002).

Los humedales son ecosistemas en constante relación con masas de agua donde se encuentran plantas adaptadas a estas condiciones (Mitsch y Gosselink, 1993). Los humedales son considerados como ecosistemas de gran fragilidad asociada a causas naturales como el cambio climático, las sequías prolongadas en la puna y a la intervención humana, como en los casos de la agricultura, ganadería, el pastoreo excesivo y la minería a cielo abierto (García, Torres, \& García, 2015). Una dimensión de la vulnerabilidad de los humedales altoandinos al cambio climático ha sido añadida por las acciones humanas y tiene que ver con los cambios en el balance hídrico en las cuencas de captación (Franco-Vidal, Delgado, \& Andrade, 2013).

Los humedales al ser alterados por cambios en el régimen hidrológico, exceso de sedimentos y contaminación, facilitan la expansión de especies invasoras, y a la vez con su biomasa de raíces contribuyen a incrementar la sedimentación (Vargas \& Díaz, 2012). Las coberturas naturales de páramo y bosque altoandino son las que mejor contribuyen a regular el ciclo hídrico (Bruijnzeel, 2004), y por ende, favorecen una gestión de adaptación al clima cambiante (Franco, Delgado, \& Andrade, 2013). En las cuencas de captación, la conversión de bosques altoandinos a pastos y las plantaciones forestales con pinos, eucaliptos y acacias, reducen los niveles de retención y almacenamiento de agua, generando la degradación de suelos (Franco et al., 2013).

Muchos humedales de montaña se han degradado por el mal manejo y desconocimiento de su importancia económica y ecológica (García et al., 2015). En los humedales del páramo de Rabanal se evidencian plantaciones de pinos, con una extensión aproximada de 1.031,59 ha (IAvH et al., 2008), se producen fuertes cambios en las propiedades químicas de los suelos, porque el crecimiento de las plantas, que implica la extracción de nutrientes, altera las propiedades químicas de estos, debido a la pérdida masiva de elementos y la acidificación del medio por la hojarasca del eucalipto y del pino principalmente, igualmente las propiedades hidrofísicas también son alteradas por la elevada evapotranspiración (Fernández et al., 2019).

Los humedales altoandinos desempeñan una función vital en el desarrollo de las cuencas andinas, así como de otros sistemas hidrográficos, ya que sus aguas fluyen hacia las vertientes de la Amazonía y hacia las costas del Pacífico y el Caribe, estos humedales y complejos de humedales mantienen una diversidad biológica única y se caracterizan por un alto nivel de endemismo de plantas y animales (García et al., 2015). Dada la importancia de estos ecosistemas, el Complejo Rabanal y Rio Bogotá cumple una función de abastecer agua que beneficia a cerca de 300 mil personas y nutre 92 acueductos locales, genera el recurso para la principal productora de energía eléctrica del país: el embalse de La Esmeralda (Central Hidroeléctrica de Chivor), e irriga más de un millón de hectáreas a través de los embalses de Gachaneca I y II (IAvH et al., 2008). 
El embalse Teatinos abastece a la ciudad de Tunja y ocupa un área de 10'740.000 metros cúbicos (Proactiva, 2014), el embalse Gachaneca I tiene una capacidad de 4'720.000 metros cúbicos; el embalse Gachaneca II ocupa 28,5 hectáreas, con una capacidad de 1'495.000 metros cúbicos (Espinosa y Espinosa, 2017), estos embalses son indispensables para la economía y seguridad alimentaria de la región. Los embalses de alta montaña como el sistema Gachanecas está rodeado de vegetación nativa como puyas, pajonales, frailejones (IAvH et al., 2008), típicos del páramo que cumplen la función de condensar el vapor y la lluvia horizontal que se da cuando el aire cargado de agua choca con los riscos y la vegetación. Por escorrentía y acumulación en turberas y en los suelos va a abastecer los embalses que sostienen la actividad de agricultura y minería en el municipio, en las épocas de baja disponibilidad, lo que hace que sea esencial mantener el sistema de páramo y alta montaña y fortalecer iniciativas para restaurar zonas que han sido degradadas por diversas intervenciones humanas (Espinosa y Espinosa, 2017).

Sin embargo, el páramo de Rabanal está amenazado principalmente por los largos procesos de intervención humana que, desde épocas precolombinas, han transformado su paisaje en un complejo mosaico socioecológico. Las coberturas vegetales y las formas de uso del suelo ponen en evidencia las distintas condiciones biofísicas y la diversidad de sistemas productivos desplegados por los habitantes, las empresas privadas y las instituciones públicas que han confluido allí a lo largo del tiempo (Fernández et al., 2019). Los principales objetivos del estudio fueron: a) identificar la influencia de la vegetación vascular en la estabilidad de los humedales artificiales del páramo Rabanal, b) Caracterizar la estructura y composición de la flora vascular en los humedales artificiales del páramo Rabanal, c) Elaborar un catálogo descriptivo de la flora vascular en estructura y composición en los humedales artificiales del páramo Rabanal.

\section{MATERIALES Y MÉTODOS}

El estudio se llevó a cabo en el denominado Complejo Rabanal y Rio Bogotá, abarca un área de 24.650,064 hectáreas, ubicado en la frontera entre los departamentos de Cundinamarca y Boyacá en un sector central de la cordillera oriental de Colombia a $5^{\circ} \mathrm{N}$ y $73^{\circ}$ 0 , específicamente en las zonas altas pertenecientes a los municipios de Ventaquemada y Samacá en el departamento de Boyacá, en las zonas circundantes a los embalses Teatinos, Gachaneca I y Gachaneca II (Figura 1) (Tabla suplementaria 1). Los muestreos se realizaron en cinco salidas de campo durante febrero y abril de 2018, se empleó un muestreo anidado (Stohlgren et al., 1995), este muestreo permite realizar mediciones de la diversidad de especies y comparar diferentes comunidades vegetales, en esencia este diseño consiste en la ubicación de manera anidada de cuadrantes (subparcelas) de $100 \mathrm{~m}^{2}$, $10 \mathrm{~m}^{2}$ y $1 \mathrm{~m}^{2}$ sucesivamente, dentro de uno mayor de $1000 \mathrm{~m}^{2}$ (Figura 2) (González-Oliva et al., 2017), dependiendo de las características fisonómicas de la vegetación (Tabla 1), se escogió un tamaño de levantamiento particular (modificado de Rangel y Velásquez, 1997) si la vegetación corresponde a un solo estrato, se empleó el área de muestreo que correspondía a esa fisonomía. En el área de estudio se realizaron 32 parcelas con un área total de $1277 \mathrm{~m}^{2}, 4$ parcelas para el estrato arbóreo, 15 para el estrato arbustivo y 13 para el estrato herbáceo. 


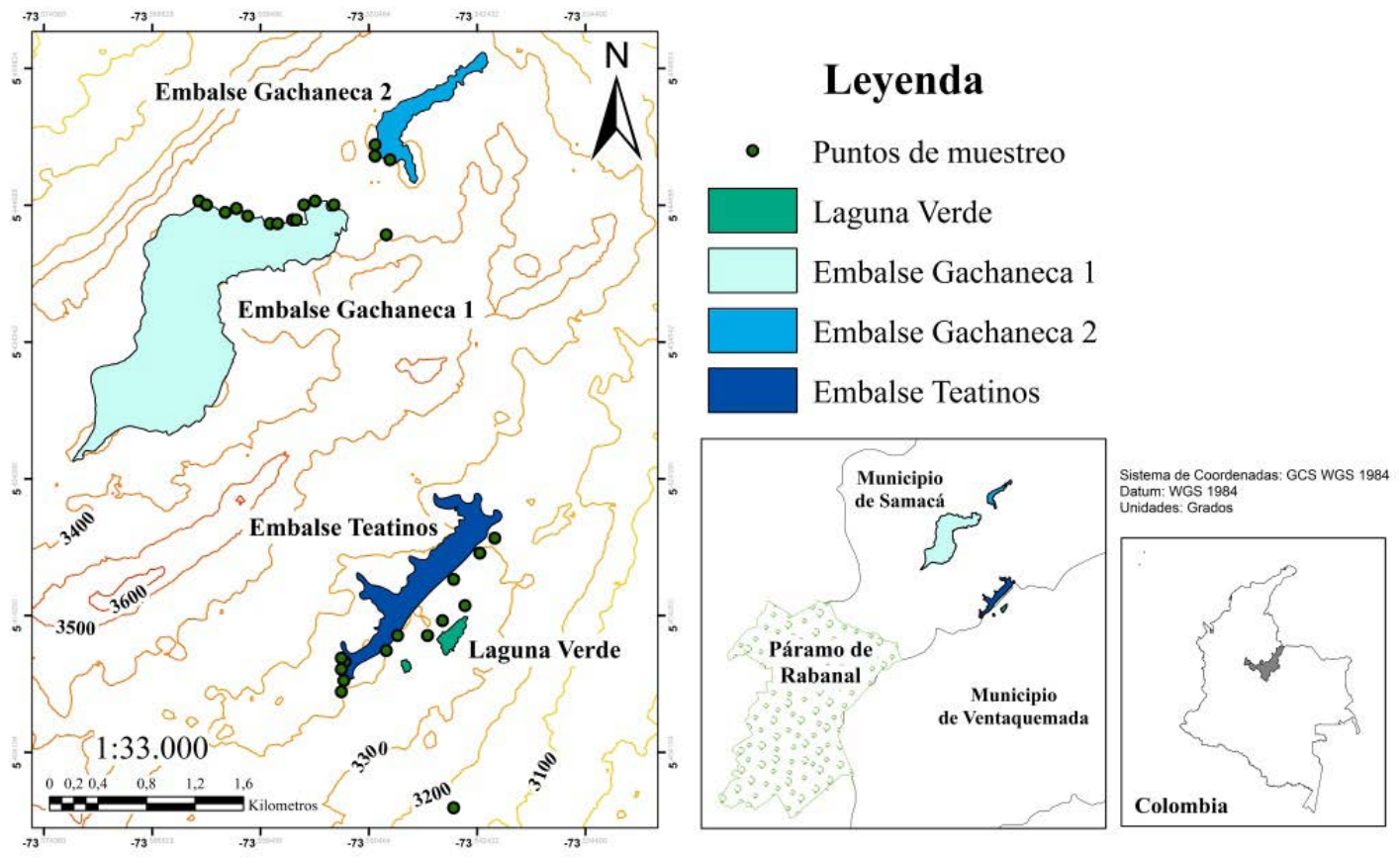

Figura 1. Localización del área de estudio: Ubicación de las parcelas en los embalses Teatinos, Gachaneca I Y Gachaneca II.

Fuente: Autores.

Tabla 1. Estratos de las características fisonómicas de la vegetación

\begin{tabular}{l|l|l} 
Estrato & \multicolumn{1}{c}{ Levantamientos } & \multicolumn{1}{c}{ Altura } \\
\hline Rasante & $1 \times 1 \mathrm{~m}$ & $0-30 \mathrm{~cm}$ \\
\hline Herbáceo & $2 \times 2 \mathrm{~m}$ & $>0,30-1,5$ \\
\hline Arbustivo & $5 \times 5 \mathrm{~m}$ & $>1,5-5 \mathrm{~m}$ \\
\hline Arbóreo inferior & $10 \times 10 \mathrm{~m}$ & $>5-12 \mathrm{~m}$ \\
\hline Arbóreo superior & $10 \times 25 \mathrm{~m}$ & $<12 \mathrm{~m}$ \\
\hline
\end{tabular}

Fuente: Autores.

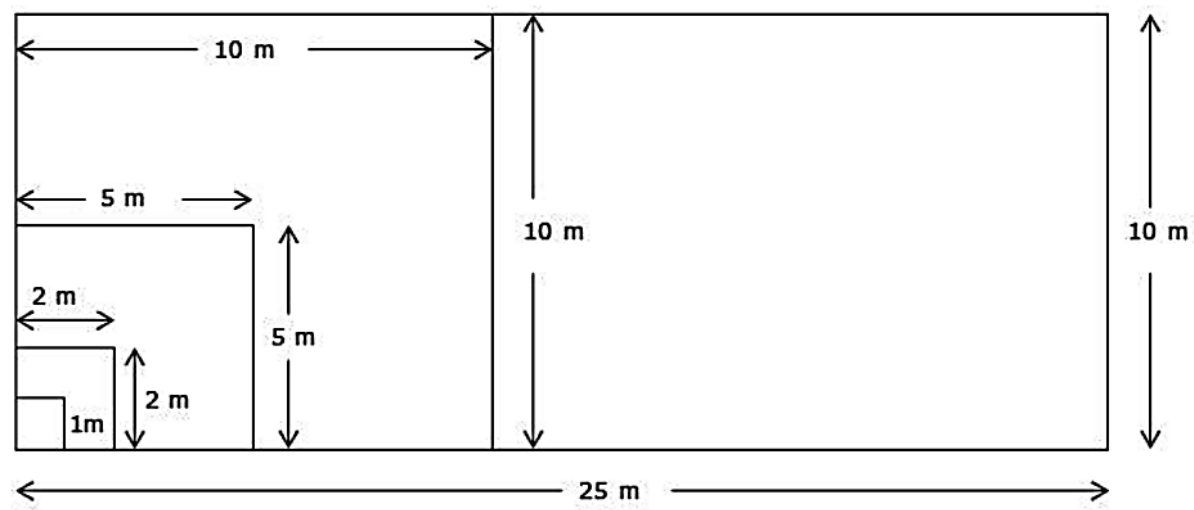

Figura 2. Diseño anidado de los levantamientos de acuerdo con la fisionomía de la vegetación.

Fuente: Autores. 
Recolección de datos y análisis de la información: se censaron todas las plantas vasculares en las 32 parcelas, en un área total de 1277 $\mathrm{m}^{2}$, se registró la información de cada individuo (nombre común, familia, género, especie, número de individuos, cobertura, estrato). Para el análisis de la diversidad alfa se aplicó el índice de dominancia de Simpson, índice de riqueza de Margalef y de diversidad de Shannon- Weaver y para la diversidad beta se determinó el índice similaridad de Sörensen para los tres embalses (Anexo 2). Los ejemplares recolectados fueron prensados y se trasladaron al Herbario de la Universidad Pedagógica y Tecnológica de Colombia (UPTC), la determinación se realizó con el apoyo de claves taxonómicas, en colaboración con el Magister Manuel Galvis Rueda y colecciones en línea (Herbario virtual UNAL y herbario virtual Jardín Botánico de Bogotá JBB).

\section{Análisis estadístico}

Se estimó la completitud del muestreo mediante el Estimador de Cobertura propuesto por Jost y colaboradores (2006). Para la elaboración de los índices de diversidad alfa y beta se empleó el programa PAST Statistics 18, la diversidad en términos de riqueza fue comparada mediante un Análisis de Varianza (ANOVA) en rangos o prueba de Kruskall-Wallis dado que los datos no cumplieron supuestos de normalidad y homocedasticidad, posteriormente se realizaron pruebas post hoc para determinar las diferencias significativas entre los embalses. Se obtuvo el índice de similitud de Bray-Curtis con el fin de evaluar la semejanza en términos de diversidad entre los embalses, cuyo valor varía entre 0 (semejanza nula) y 1 (100\% de semejanza).

\section{RESULTADOS Y DISCUSIÓN}

Se censaron 2220 individuos en las 32 parcelas, con sus diferentes estratos dependiendo de las características fisonómicas de la vegetación, las familias con mayor número de géneros para los tres embalses son Asteraceae, Poaceae, Ericaceae, Melastomataceae y Rosaceae (Tabla 2) (Figura 3). Igualmente, las familias con mayor número de especies para los embalses fueron Eriocaulaceae, Ericaceae, Poaceae, Bromeliaceae, Cyperaceae, Asteraceae, Rubiaceae, Lycopodiaceae, Cunoniaceae y Juncaceae (Figura 4), todos los ejemplares correspondían a plantas vasculares presentes en las zonas circundantes a los humedales artificiales del páramo Rabanal

Tabla 2. Número de familias, géneros y especies en los diferentes estratos de vegetación.

\begin{tabular}{|c|c|c|c|c|c|c|c|}
\hline \multirow[b]{2}{*}{ Embalse } & \multirow[b]{2}{*}{ Familias } & \multirow[b]{2}{*}{ Géneros } & \multirow[b]{2}{*}{ Especies } & \multicolumn{4}{|c|}{ Estrato (Número de sp) } \\
\hline & & & & Rasante & Herbáceo & Arbustivo & Arbóreo \\
\hline Teatinos & 36 & 84 & 64 & 10 & 45 & 25 & 3 \\
\hline Gachaneca I & 19 & 25 & 26 & 1 & 15 & 8 & 2 \\
\hline Gachaneca II & 30 & 45 & 50 & 1 & 29 & 16 & 5 \\
\hline
\end{tabular}

Fuente: Autores. 


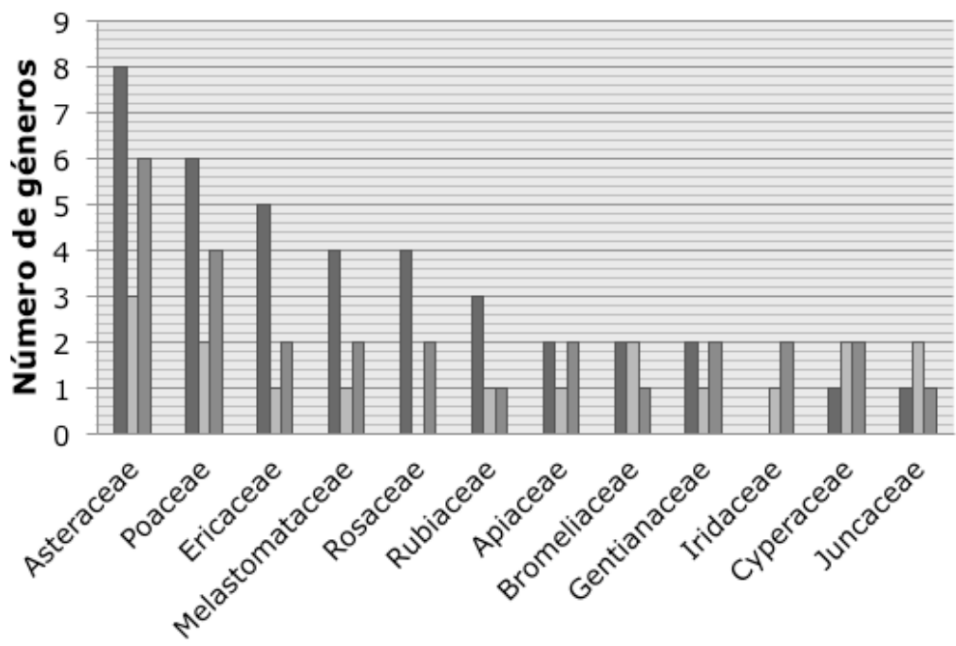

Embalse Teatinos

$\square$ Embalse Gachaneca I

Embalse Gachaneca II

Figura 3. Familias con mayor número de géneros en los embalses Teatinos, Gachaneca I y Gachaneca II.

Fuente: Autores.
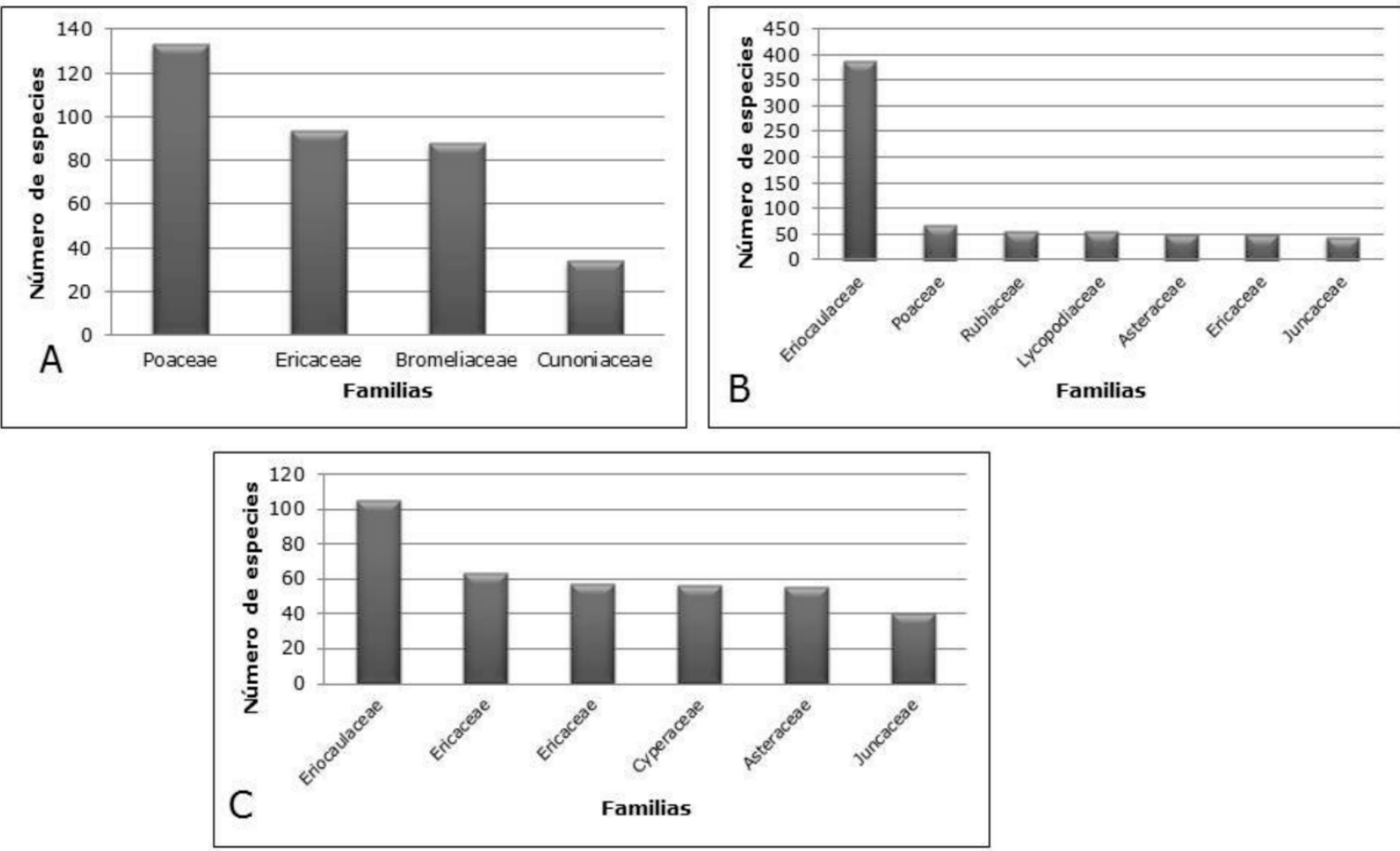

Figura 4. Familias con mayor número de especies. A. Embalse Teatinos. B. Embalse Gachaneca I. C. Embalse Gachaneca II.

Fuente: Autores. 
Las especies de plantas vasculares que predominaron por su mayor número de individuos para el embalse Teatinos en el estrato arbóreo: Weinmannia tomentosa L.f. (34), para el estrato arbustivo Chusquea Scandens Kunth (133), estrato herbáceo Calamagrostis effusa (Kunth) Steud (160) y estrato rasante Nertera granadensis (Mutis ex L. f.) Druce (15), para el embalse Gachaneca I en el estrato arbóreo Weinmannia tomentosa L.f. y Bucquetia glutinosa (L. f.) DC. (1), estrato arbustivo Arcytophyllum muticum (Wedd.) Standl (55), estrato herbáceo Paepalanthus columbiensis Ruhland (389) y estrato rasante Geranium multipartitum Benth (6), finalmente para el embalse Gachaneca II en el estrato arbóreo: Pinus patula Schltdl. \& Cham. (35), estrato arbustivo Pernettya prostrata (Cav.) DC. (63), estrato herbáceo Paepalanthus columbiensis Ruhland (105) y estrato rasante Paspalum bomplandianum Flüggé (16) (Anexo 1).

El número de especies de plantas vasculares registradas para el páramo Rabanal en cada uno de los embalses representa una muestra significativa en la región paramuna del departamento de Boyacá. El Estimador de Cobertura (Jost, 2006) indica que el esfuerzo de muestreo fue suficiente con valores por encima del 99 $\%$ de representatividad para los tres embalses (Gachaneca I = 0.998; Gachaneca II = 1; Teatinos $=0.99)$, indicando una nula probabilidad de encontrar más especies si se aumenta el esfuerzo de muestreo, o si se registran más individuos (Figura 5).

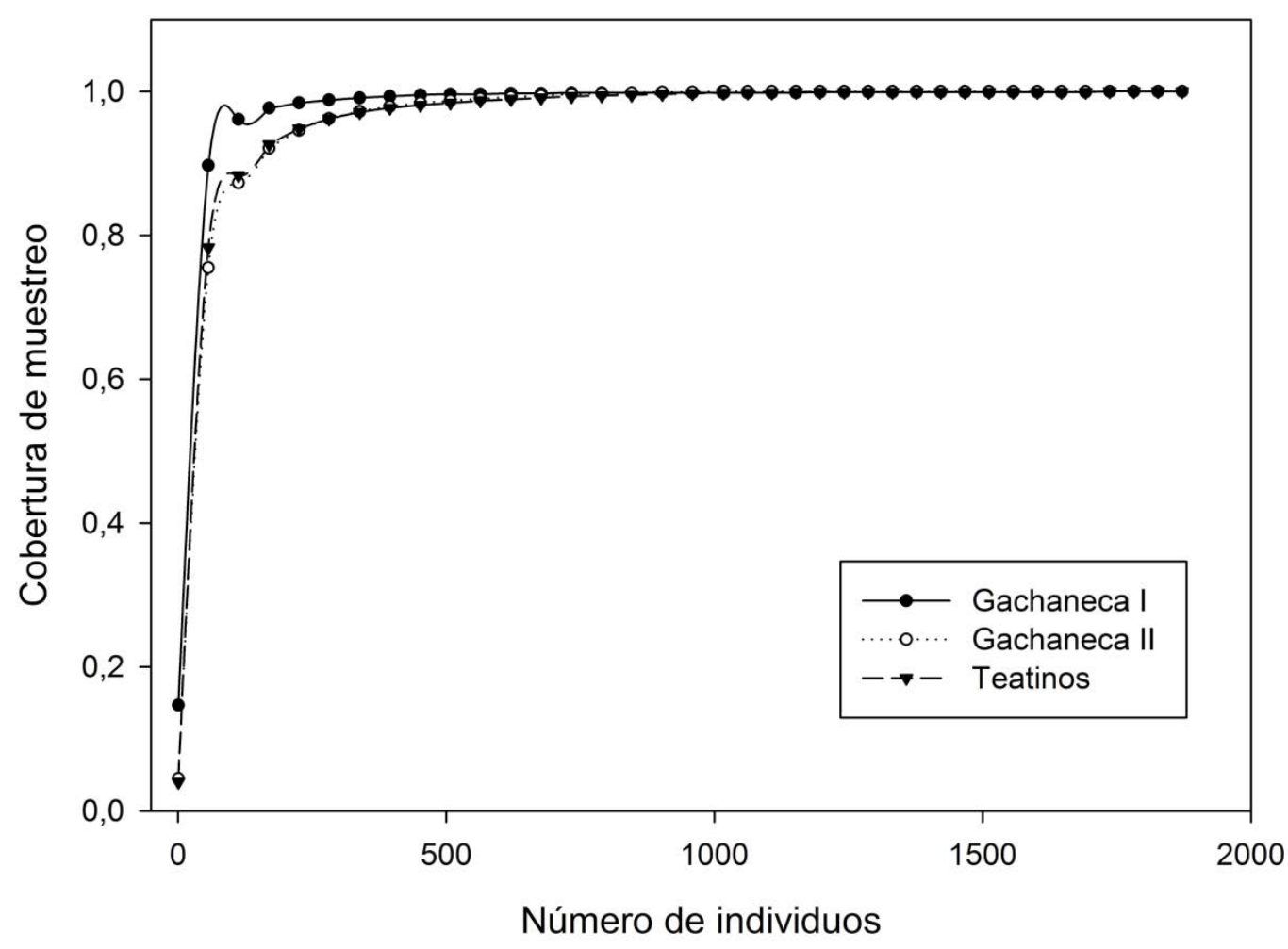

Figura 5. Curva de cobertura de muestreo para los 3 embalses con respecto al número de individuos.

Fuente: Autores. 
El análisis de varianza evidenció diferencias estadísticas entre las diversidades en términos de riqueza. La diversidad de Teatinos fue significativamente diferente de la de Gachaneca I y II $(p=0.002 ; p=0.001)$, por su parte Gachaneca I y Gachaneca II no tuvieron diferencias $(p=0.067)$. El índice de Bray-Curtis de igual manera arroja una mayor similitud en estructura y composición de la diversidad entre Gachaneca I y II con 53 \% de semejanza, siendo Teatinos el embalse más disímil con solo un 32 $\%$ de semejanza con los demás (Figura 6).

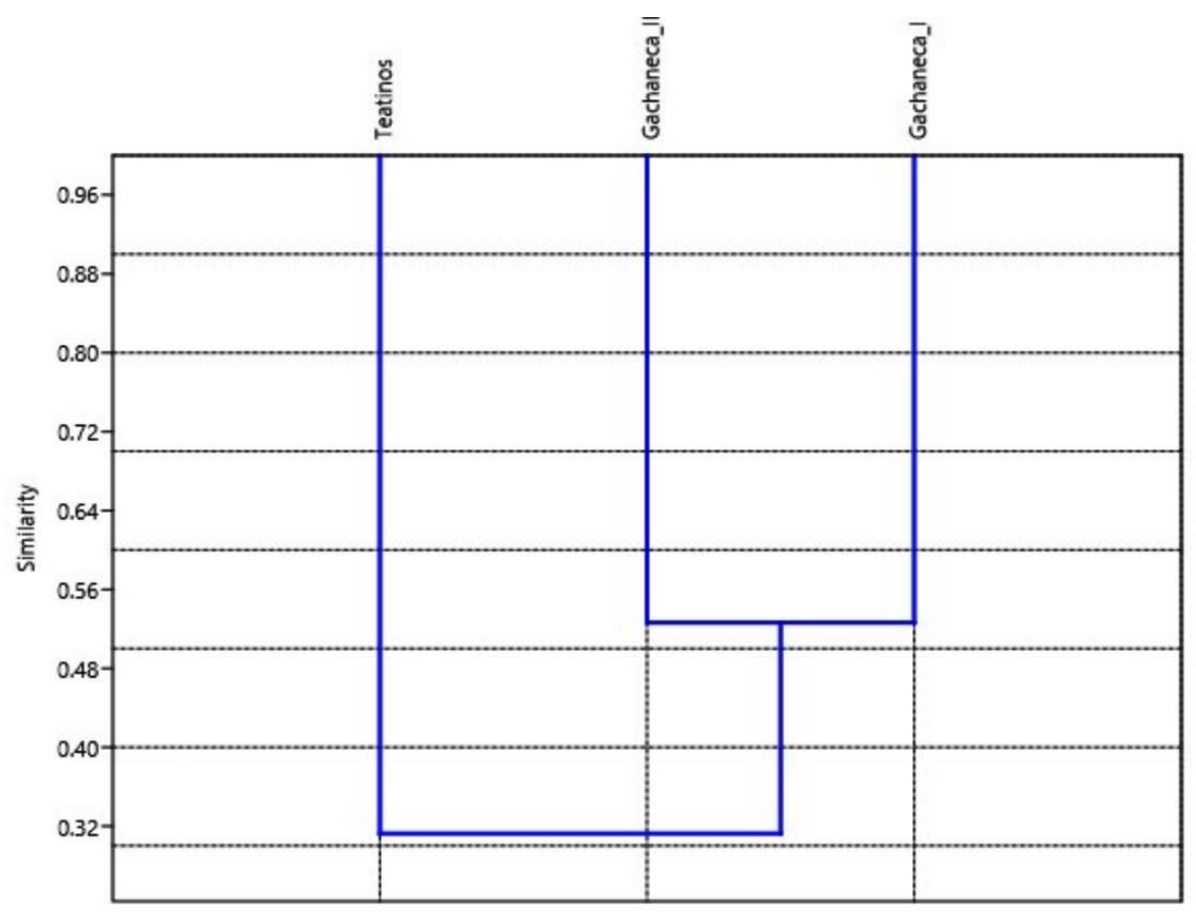

Figura 6. Similitud de Bray-Curtis entre los embalses evaluados.

Fuente: Autores.

En la lista de plantas referenciada en Capacho (2016) para el paramo de Almorzadero las familias mas ricas en generos, especies y morfoespecies son Asteraceae, Poaceae y Cyperaceae, los géneros mas ricos en especies y morfoespecies son Lachemilla, Hyperycum, Carex y Senecio, al comparar estos resultados con los obtenidos del presente estudio, se evidencia que hay coincidencia en el registro de 12 familias, 13 generos y 10 especies.

Cetina (2018) identificó las comunidades vegetales en un área de restauración ecológica de bosque altoandino, en el Parque Forestal Embalse del Neusa en Cundinamarca, determino 32 especies, 27 géneros de 19 familias, 
en el cual, la familia más diversa corresponde a Asteraceae (7 especies), seguida por Hypericaceae (3), Poaceae (3) y Rosaceae (3), lo cual coincide con el presente estudio en 15 familias, 21 generos y 14 especies, el estrato herbáceo es el mas respresentativo equivalente a $42 \%$ y el menor valor corresponde al estrato rasante con un $7 \%$, lo cual coincide con el presente estudio. Igualmente, para los estratos de vegetacion el mayor valor es para el herbaceo con un valor de $56 \%$, sin embargo, el menor valor se presentó para el estato arbóreo con un $6 \%$ (Figura 7), al comparar estos resultados se observa que los embalses del paramo Rabanal presentan mayor riqueza de especies, se precisa una diferencia de 64 especies.

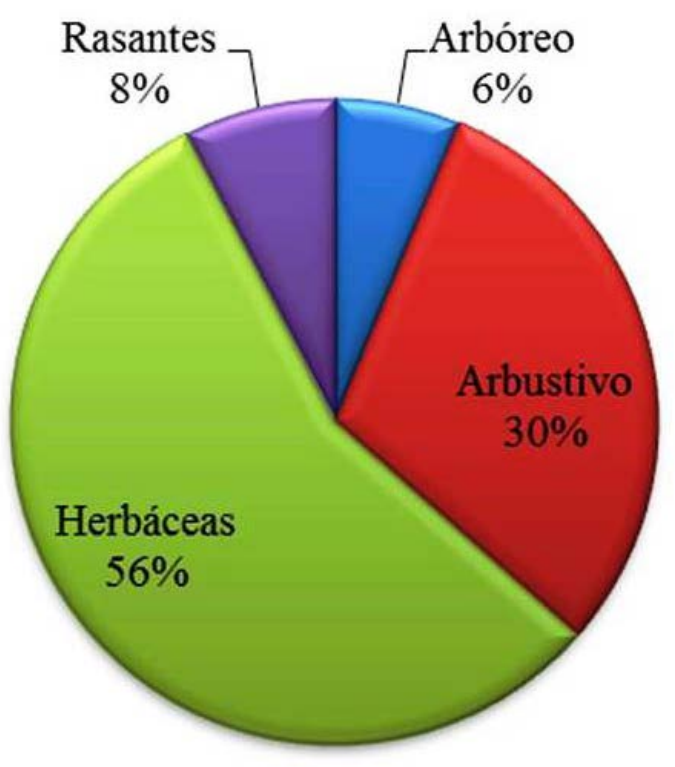

Figura 7. Distribución general de las especies según el estrato.

Fuente: Autores.

Según los valores del índice de Margalef, se consideró que el área estudiada presenta una riqueza alta, la parcela $\mathrm{P} 2$ registro el mayor valor de riqueza de especies $(\mathrm{Mg}=5,40)$, seguida de la parcela P1 $(4,94)$, en tanto que los menores valores se encontraron en las parcelas P15 y P22 con 1,31 y 1,42, respectivamente. Los valores de diversidad más altos según el índice de Shannon-Wiener correspondieron a la parcela $\mathrm{P} 2\left(\mathrm{H}^{`}=4,39\right), \mathrm{P} 1 \quad\left(\mathrm{H}^{`}=4,21\right)$ y $\mathrm{P} 19$ $\left(H^{`}=4,00\right)$, en tanto que el más bajo se registró en la parcela P15, P22, P30 y P12 $\left(\mathrm{H}^{`}=2,23\right.$; $\mathrm{H}^{`}=2,26 ; \mathrm{H}^{`}=2,41$ y $\mathrm{H}^{`}=2,43$, respectivamente). Según los valores obtenidos en el índice de Simpson, las parcelas P2, P1, P5, P19 presentaron mayor dominancia $(\lambda=0,94 ; \lambda=0,93$; $\lambda=0,92$ y $\lambda=0,92)$, la menor dominancia correspondió a la parcela P3 $(\lambda=0,50)$ (Tabla 3). 
Tabla 3. Índices de diversidad alfa

\begin{tabular}{|c|c|c|c|}
\hline Numero de parcela & Índice de Simpson & Índice de Shannon-Weaver & Índice de Margalef \\
\hline 1 & 0,94 & 4,22 & 4,94 \\
\hline 2 & 0,95 & 4,39 & 5,40 \\
\hline 3 & 0,50 & 3,78 & 3,09 \\
\hline 4 & 0,91 & 3,67 & 3,01 \\
\hline 5 & 0,93 & 3,88 & 3,10 \\
\hline 6 & 0,88 & 3,22 & 2,46 \\
\hline 7 & 0,88 & 3,64 & 4,23 \\
\hline 8 & 0,77 & 3,00 & 3,19 \\
\hline 9 & 0,71 & 3,81 & 2,44 \\
\hline 10 & 0,88 & 3,39 & 2,98 \\
\hline 11 & 0,85 & 3,05 & 2,04 \\
\hline 12 & 0,66 & 2,43 & 2,13 \\
\hline 13 & 0,81 & 3,05 & 2,64 \\
\hline 14 & 0,73 & 2,71 & 2,40 \\
\hline 15 & 0,77 & 2,24 & 1,31 \\
\hline 16 & 0,85 & 2,99 & 1,87 \\
\hline 17 & 0,78 & 2,52 & 1,56 \\
\hline 18 & 0,87 & 3,14 & 2,50 \\
\hline 19 & 0,92 & 4,00 & 4,32 \\
\hline 20 & 0,85 & 3,17 & 2,38 \\
\hline 21 & 0,86 & 3,12 & 2,72 \\
\hline 22 & 0,75 & 2,27 & 1,43 \\
\hline 23 & 0,90 & 3,54 & 2,60 \\
\hline 24 & 0,87 & 3,22 & 1,97 \\
\hline 25 & 0,89 & 3,40 & 2,44 \\
\hline 26 & 0,89 & 3,39 & 2,37 \\
\hline 27 & 0,86 & 3,12 & 2,19 \\
\hline 28 & 0,85 & 2,99 & 2,40 \\
\hline 29 & 0,89 & 3,33 & 2,04 \\
\hline 30 & 0,77 & 2,42 & 1,88 \\
\hline 31 & 0,75 & 2,54 & 2,20 \\
\hline 32 & 0,80 & 2,65 & 2,11 \\
\hline
\end{tabular}

Fuente: Autores. 
En cuanto al índice de Shannon-Weaver, los resultados de este trabajo mostraron que 22 de las 32 parcelas tuvieron valores mayores de 3, lo que indica una heterogeneidad de comunidad vegetal alta (Caviedes, 1999). Igualmente, para el índice de Simpson 30 parcelas presentan una diversidad alta, en general para los embalses Teatinos, Gachaneca I y Gachaneca II presentaron una dominancia baja de especies y una diversidad alta. Los valores del índice de Margalef arrojados muestran que la Parcela P2 obtuvo un valor de 5,40 que presenta una diversidad alta, en contraste con 6 parcelas con valores menores a dos con baja diversidad (tabla 3). Al emplear el índice de similaridad de Sörensen para los tres embalses se observa que los mayores valores corresponden a los embalses Gachaneca I y II, poseen un $52,63 \%$ de similaridad de las comunidades vegetales y el menor valor para el embalse Teatinos y Gachaneca I con un $36,69 \%$ (tabla 4 ).

Tabla 4. Índice de diversidad beta, índice de similaridad de Sörensen para los tres embalses.

\begin{tabular}{l|c|c} 
Embalse 1 (Comunidad A) & Embalse II (Comunidad B) & Índice de Sörensen \\
\hline Teatinos & Gachaneca I & $36,69 \%$ \\
\hline Teatinos & Gachaneca II & $51,12 \%$ \\
\hline Gachaneca I & Gachaneca II & $52,63 \%$ \\
\hline
\end{tabular}

Fuente: Autores.

La caracterización de las especies indicó que los embalses del páramo Rabanal, están siendo afectados por el establecimiento de especies invasoras y colonizadoras como Acacia melanoxylon R. Br., Lachemilla orbiculata (Ruiz \& Pav.) Rydb., Pinus patula Schltdl. \& Cham., Rumex acetosella L. y Anthoxantum odoratum L. que pueden remplazar las especies nativas y transformar el hábitat. La implantación de árboles exóticos en el páramo afecta la estabilidad del ecosistema, especialmente especies como el pino y eucalipto consumen abundante agua, disminuyen el rendimiento hídrico y restringen la disponibilidad de agua en el suelo formando más descomposición (Gomezcoello, 2020). Las plantaciones de pinos en páramos tienen una evapotranspiración mayor y una escorrentía menor (García y Torres, 2019), ocasionando asi, una reducción en la productividad hídrica y en la cobertura vegetal natural.

Las plantaciones de pino disminuyen el valor ecológico de los ecosistemas naturales del ecosistema paramo, las sustancias resinosas exudadas por los pinos dificultan el humedecimiento del suelo, bloqueando los poros; debido a los valores altos de porosidad, baja densidad aparente y poca capacidad de retención de humedad. Buytaert et al. (2007) demostraron en un estudio de cuencas comparadas que las plantaciones de pino pueden reducir el rendimiento hídrico del páramo hasta en un $60 \%$, más aún que los cultivos agrícolas. En un área aproximada de 1.000 ha de pino sembrada para explotación privada en el páramo de Rabanal, las condiciones edáficas no están siendo aprovechadas de la mejor manera para la acumulación de agua, lo cual es grave, ya que este páramo provee del preciado líquido a cerca de 300.000 personas en Tunja y algunas poblaciones aledañas (Fernández et al., 2019).

\section{CONCLUSIONES}

La caracterización de la vegetación vascular se constituye en una herramienta fundamental para el desarrollo de estrategias de 
restauración en la búsqueda del equilibrio de los ecosistemas disturbados (Rodríguez-Lombana, Beltrán-Gutiérrez, \& Moreno, 2017). La flora vascular de los humedales artificiales presentó un patrón similar a estudios realizados en diversos humedales en relación a las familias más dominantes como lo son Poaceae, Cyperaceae y Asteraceae, se evidenció que el estrato herbáceo es el más dominante presente en los alrededores de los embalses.

El ecosistema de paramo brinda varios servicios ecosistémicos de vital importancia para la población humana, por tal razón, es de extrema urgencia implementar estrategias que permitan la conservación del páramo, la degradación de estos conlleva a una perdida elevada a nivel económico, social y ecológico, se debería mantener la riqueza y el potencial inmenso que se puede obtener a través de los servicios ambientales y manejo sustentable que brinda el páramo, como son el agua para riego, para consumo humano, electricidad y uso diario (Padilla, 2014).

Existen diferencias significativas de diversidad entre los tres embalses, los embalses Gachaneca I y Gachaneca II son más parecidos en diversidad y composición que con el embalse Teatinos.

La plantación de pinos que se muestrearon en el páramo Rabanal afecta directamente las cuencas de captación, puesto que sus raíces absorben gran cantidad de agua, perjudicando directamente a la población aledaña que se beneficia de este recurso.

\section{CONTRIBUCIÓN DE LA AUTORÍA}

Todos los autores han realizado colectivamente la investigación, metodología, trabajo de campo, redacción, análisis de datos, determinación del material vegetal, conceptualización, escritura, revisión y edición.

\section{AGRADECIMIENTOS}

Los autores expresan su agradecimiento a la Universidad Pedagógica y Tecnológica, a la escuela de la Maestría en Ciencias Biológicas por su acompañamiento, al Herbario (UPTC) por facilitar el secado, montaje y determinación del material recolectado.

\section{LITERATURA CITADA}

Buytaert, W., Iñiguez, V., y Bièvre, B. D. (2007). The effects of afforestation and cultivation on water yield in the Andean páramo. Forest Ecology and Management, 25(1), 22-30. https://doi. org/10.1016/j.foreco.2007.06.035

Bruijnzeel, L. A. Sampurno. (2004). Hydrological functions of tropical forests: Not Seeing the Soil for the Trees? Agriculture, Ecosystems \& Environment, 104(1), 185-228. https://doi.org/10.1016/j. agee.2004.01.015

Capacho, D. I. (2016). La vegetación de los humedales altoandinos del sector de Presidente, páramo de Almorzadero (Norte de Santander, Colombia) (Tesis de Doctorado). Universidad de Alicante, España.

Corporación Autónoma Regional de Cundinamarca (CAR) y Corporación Autónoma de Chivor (CORPOCHIVOR). (2002). Conservación y manejo sostenible de los páramos Cristales, Castillejo, cuchilla el Choque y nacimiento río Bogotá. Versión digital. Bogotá, D. C., Colombia. Recuperado de: http://sie.car.gov.co/handle/20.500.11786/35709

Caviedes, B. M. (1999). Manual de métodos y procedimientos estadísticos. Universidad Jorge Tadeo Lozano. Bogotá, Colombia.

Cetina, A. (2018). Comunidades vegetales de páramo presentes en un área de restauración ecológica de bosque altoandino, Parque Forestal Embalse del Neusa (Cundinamarca-Colombia). Pontificia Universidad Javeriana. Recuperado de: https://repository.javeriana.edu.co/handle/10554/40227

Cleef A. M. (1978). Characteristics of neotropical paramo vegetation and its subantartic relations. In: Troll, C. \& Lauer, W. (eds.), Geological relations between the souththern temperate zone and the tropical mountain. (pp. 365-390). Erdwiss Forsch. Wiesbaden, Germany. 
Cuatrecasas, J. (1989). Aspectos de la vegetación natural de Colombia. Pérez- Arbelaezia 2(8): 155-283.

Espinosa, J. S., y Espinosa, A. J. (2017). Problemática ambiental Paramo de Rabanal en Samacá: Visión desde la asociación de usuarios del distrito de adecuación de tierras de Samacá (ASUSA). Universidad pedagógica y Tecnológica de Colombia (UPTC). Recuperado de: https://www.researchgate.net/ publication/327977614_PROBLEMATICA_AMBIENTAL_PARAMO_DE_RABANAL_EN_SAMACA VISION_DESDE_LA_ASOCIACION_DE_USUARIOS_DEL_DISTRITO_DE_ADECUACION_DE_ TIERRAS DE SAMACA ASUSA

Etter, A., McAlpine, C., y Possingham, H. (2008). Historical patterns and drivers of landscape change in colombia since 1500: A regionalized spatial approach. Annals of the Association of American Geographers, 98(1), 2-23. https://doi. org/10.1080/00045600701733911

Fernández, C. J., Cely, G. E., y Serrano, P. A. (2019). Cuantificación de la captura de carbono y análisis de las propiedades del suelo en coberturas naturales y una plantación de pino en el páramo de Rabanal, Colombia. Revista Colombiana de Geografía, (28), 121-133. https://doi.org/10.15446/ rcdg.v28n1.66152

Franco-Vidal, L., Delgado, J., y Andrade, G. (2013). Factores de la vulnerabilidad de los humedales altoandinos de Colombia al cambio climático global. Revista Colombiana de Geografia, 22(2), 6985. https://doi.org/10.15446/rcdg.v22n2.37018

García, M. (2019). Estudio comparativo entre vegetación de páramo conservado e intervenido por plantación de pino, Cotopaxi-Ecuador (tesis de pregrado). Universidad Central de Ecuador, Quito, Ecuador. Recuperado de: http://www.dspace. uce.edu.ec/handle/25000/19328

García, J. E., Torres, J., y García, F. T. (2015). Identificación de especies en los humedales altoandinos de la concesión para la Conservación Alto Huayabamba. Scientia, 7(1), 13-18.

Gomezcoello, C. A. (2020). Resiliencia de la microcuenca del río Matadero frente a riesgos exógenos (tesis de pregrado). Universidad de Cuenca, Cuenca, Ecuador. Recuperado de: http://dspace. ucuenca.edu.ec/handle/123456789/34106

González-Oliva, L., Ferro-Díaz, Rodríguez-Cala , J., D. y Berazaín., R. (2017). Métodos de inventario de plantas. En C. A. Mancina y D. D. Cruz. (Ed.), Diversidad biológica de Cuba: métodos de inventario, monitoreo y colecciones biológicas ( $p$. 60-85). Habana, Cuba: Editorial AMA, La Habana. Recuperado de: https://www.latinamerica.undp. org/content/rblac/es/home/library/poverty/-diversidad-biologica-de-cuba--metodos-de-inventario--monitoreo-.html

Instituto Von Humbold (IAvH), Corporación Autónoma Regional de Cundinamarca (CAR) Corporación Autónoma Regional de Boyacá (CORPOBOYACÁ) y Corporación Autónoma de Chivor (CORPOCHIVOR). (2008). Estudio sobre el estado actual del macizo del páramo de Rabanal Convenio interadministrativo No. 07-06-263-048 (000404). Recuperado de: https://fauna.corpochivor.gov.co/ wp-content/uploads/2016/04/Estudio-del-estado-actual-del-paramo-de-Rabanal.pdf

Jost, L. (2006). Entropy and diversity. Opinion, $113(2), 363-375$.

Luteyn, J. L. (1999). Páramos: A check list of plant diversity, geographical distribution and botanical literature. Memoirs of the New York Botanical Garden (84), 1-278.

Mitsch, W. J. y Gosselink, J. G. (1993). Wetlands, 2nd Edition. Van Nostrand Reinhold Co., New York, NY, USA. 1-722.

Molano, J. (1989). Biogeografía de los páramos de Colombia. Revista de la Sociedad Colombiana de la Ciencia del Suelo, 19(1), 5-10.

Padilla, M. (2014). Estudio multitemporal del uso del suelo y cobertura vegetal natural en el páramo de la parroquia Mulaló (tesis de maestría). Universidad Técnica de Ambato, Ambato, Ecuador. Recuperado de: http://repositorio.uta.edu.ec/ handle/123456789/7692

Proactiva Aguas de Tunja (2014). Análisis y formulación de la ingeniería básica de alternativas para el abastecimiento futuro de agua para la ciudad de Tunja (Informe de la Gerencia operativa y de construcciones). Tunja, Colombia. Recuperado de: https://alcaldiatunja.micolombiadigital.gov.co/sites/alcaldiatunja/content/ files/000712/35570_2019_23dic_otro_si_contrato_concesion_132_1996.PDF

Rangel-Ch, J. O., y Velázquez, A. (1997). Métodos de estudio de la vegetación. In J. O. Rangel-ch (Ed.), Colombia Diversidad Biótica II (Universidad, pp. 59-87). Santa Fé de Bogotá, Colombia: Instituto de Hidrología, Meteorología y Estudios Ambientales-IDEAM- y Academia Colombiana de Ciencias Exactas Físicas y Naturales. 
Rodríguez-Lombana, A. R., Beltrán-Gutiérrez, H. E., y Moreno, A. C. (2017). Caracterización florística del bosque subandino y algunas áreas disturbadas en San Bernardo (Cundinamarca), Colombia. Biota Colombiana, 18(2), 42-71. https://doi. org/10.21068/c2017.v18n02a04

Stohlgren, T. J., M. B. Falkner y L. D. Schell. (1995). A modified-Whittaker nested vegetation sampling method. Vegetatio 117(2), 113-121.
Tapia, C. (2014). Plan Participativo de Manejo y Conservación del Páramo de Rabanal (No. 45). Bogotá, Colombia. Recuperado de http://paramos.humboldt.org.co

Vargas, O., y Díaz, A. (2012). Primera parte. In Catálogo de plantas invasoras de los humedales de Bogotá (pp. 13-23). Bogotá, D.C. 